\title{
Fast fault location scheme for distribution systems based on fault transients
}

\author{
F. M. Aboshady*†, D. W. P. Thomas*, M. Sumner* \\ * University of Nottingham, Nottingham, UK, fathy.aboshady@nottingham.ac.uk \\ ${ }^{\dagger}$ Tanta University, Tanta, Egypt, eng.aboshady@f-eng.tanta.edu.eg
}

Keywords: Fault location; distribution system; high frequency; impedance based methods.

\begin{abstract}
This paper presents a combined double-end and single-end fault locator for distribution systems. The technique lies under the impedance based category and uses the fault generated high frequency components to locate the faults. The combination of double-end and single-end allows the method to discriminate between faults on the main feeder and those on laterals. Also, the method only requires a short data window as it depends on the high frequency components. The evaluation of the method considers different system and fault parameters e.g. loading taps, loading unbalance, fault type and fault resistance. To validate the proposed technique, the IEEE 34 nodes system is used to simulate different test cases.
\end{abstract}

\section{Introduction}

With competitive electricity markets, continuity of service is an important issue. However, the power network is continuously growing in size and complexity which increases the probability for failures leading to reduced continuity indices $[1,2]$. A reliable fault locator, which is capable of precisely identifying the faulted part of the network, can reduce the outage time and help with fast restoration. For these reasons, several fault location techniques have been reported for both transmission and distribution systems [3-6]. At the level of the distribution system, the fault location problem has been formulated based on various approaches that include fundamental frequency analysis (to find the system's apparent impedance to the fault point), detecting the Travelling Waves (TW) generated at the onset of a fault, applying artificial intelligence, and other methods that use for example distributed measurement devices such as smart meters being used in modern distribution systems [7].

Impedance based fault location methods are more promising for distribution systems compared to travelling waves and artificial intelligence methods. The impedance based methods are cheaper to implement because they usually require processing and capturing data at a lower sampling rate compared to travelling waves (which typically require a sampling rate of several $\mathrm{MHz}$ or even $\mathrm{GHz}$ [8]). Also, for application to distribution systems, TW methods need to distinguish between different waves reflected from different discontinuity points along the system which increases the method's complexity. Using artificial intelligence tools such as artificial neural networks requires extensive training and generation of data banks for all system possible configurations [9]. In a move towards more automated systems, modern distribution system have become instrumented with devices such as Intelligent Electronic Devices (IEDs), smart meters and fault indicators. Attempts to gain further benefits from these devices to help in locating faults in distribution systems have been devised [10, 11]. In [10], by using measurements at the substation along with other available IEDs on the system, the fault point is located. In [11], directional fault indicators have been employed to find the fault section instead of the exact location. In [12], voltage sag matching between actual recorded fault and simulated faults at all system nodes is used to find the closest node to the fault assuming the availability of measurements at all nodes.

In [13], an impedance based fault location method using circuit analysis of the high frequency responses generated due to the fault is used to locate faults in integrated power distribution systems. It was necessary in this method to measure at both ends of each line segment. When it was implemented on a system with a tapped loads, a very high error in the estimated fault distance was obtained. Likewise, a high sampling rate of $1 \mathrm{MHz}$ was used for data measurements. The benefit of this method is the short data window required to locate the fault. The practical execution of such methods calls for further improvements to cut down the number of measurement points and the required sampling rate. A similar concept for applying high frequency components was presented in [14]. To study the issue of tapped loads and laterals with a reduced number of measurements, 11 unsynchronised IEDs as well as measurements at the main substation was employed to locate faults in a 33 node system

In this paper, the high frequency components generated with the fault are used for fault location purposes similar to [13, 14]. Due to dependence on the high frequency components, the method requires a short window of recorded data for the fault location process, less than one cycle after the fault. This feature allows the algorithm to locate sub-cycle and temporary faults that extend to less than one cycle and use such information to modify the maintenance schedules to consider the system weak spots. Unlike [13, 14], a sampling rate of $20 \mathrm{kHz}$ is used for synchronised data measurements at only two points along the feeder which simplifies and reduces 
the cost of its real implementation. Firstly, the double-end algorithm is derived to locate faults along the main feeder. After that, the concept of a combined double-end and singleend algorithm to distinguish between fault on the main feeder and fault on a lateral will be explained.

\section{Double-end method}

The common drawback of impedance based fault location techniques based on single-end measurements is their inability to distinguish between faults at the same distance from the substation [3, 15]. Fault location techniques that consider the availability of two synchronised measurement points were introduced in $[15,16]$. In [15], a method which starts by applying a single-end method to find all candidate fault locations then moves to synchronised measurements from two points to eliminate the incorrect locations was proposed. Only faults along the main feeder were simulated in [16]. In this paper an alternative combined method is proposed which starts with applying a double-end method. In this section, the double-end method is derived. Then, the performance of this method for faults on the main feeder and those on a lateral are described.

\subsection{Double-end method derivation}

The method depends on measurements at the substation and the end node of the main radial system. In this paper, the analysis neglects the effect of the distribution line capacitance. The basic principle is illustrated considering a fault at any point on the main feeder between the two end nodes S (sending end) and R (receiving end) as shown in Fig 1. The fault can be considered to be a step voltage source behind the fault impedance and has a step change equal and opposite to the pre-fault voltage at the fault point as clear in Fig. 1. Using a simple three phase circuit analysis, the value of per unit fault distance $\mathrm{x}$ can be obtained using (1), where $\mathrm{Z}$ is the known line impedance and $\mathrm{Z}_{\mathrm{f}}$ is the fault impedance.

$$
x Z\left(I_{S}-I_{R}\right)=V_{S}-V_{R}-Z I_{R}
$$

Equation (1) is a general equation valid for different fault types. By applying (1) at different frequencies, a series of values for $\mathrm{x}$ is obtained for each phase at each frequency. Only values related to the faulted phase/s are considered. Curve fitting is applied on the frequency range up to $2 \mathrm{kHz}$ to find the final estimated fault distance.

This method can be refined to incorporate the effect of load taps by using a ladder power flow technique such as that proposed in [17]. Using measured Vs, Vr, Is and Ir, and a knowledge of the cable impedance per unit length and an estimate of tapped loads the voltages and currents at each section of line can be obtained through sweeping up and downstream. To locate a fault, the fault is firstly assumed to be in the first section next to the substation. If the estimated fault distance is greater than the total section length, the ladder algorithm is re-applied to the next section based on the first estimation until a distance less than the section length is calculated.

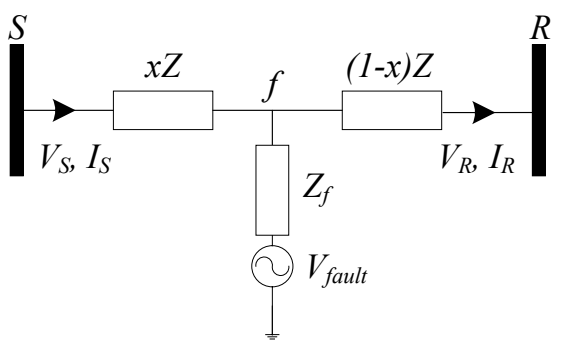

Fig. 1: Double-end method equivalent circuit

\subsection{Performance analysis for different faults}

To illustrate the benefit of the proposed combined method, the following example is used. For the system of Fig. 2, the horizontal black line with the two measurement points represents the main feeder and there are two laterals, represented by the red lines, at points $\mathrm{T} 1$ and $\mathrm{T} 2$.

Consider three fault cases, all at the same distance from the main substation. The first fault lies on the main feeder at point $\mathrm{F}$ and the other two lie on the laterals at points F1 and F2. By using the double-end method, output for the first fault will, ideally, indicate a fault at F. However, for the second and the third faults, the fault will appear to be at the tapping point $\mathrm{T} 1$ and T2 respectively. Although the lateral fault is not completely located, the method provides only one solution which is a great advantage.

Nevertheless, a new problem arises, which is discriminating between a fault on the lateral (and defining its exact location) and a fault at the tapping point: in both cases, the double-end method will indicate a fault at the tapping point. The proposed solution for this problem is the combined doubleend and single-end method which is explained in the next section.

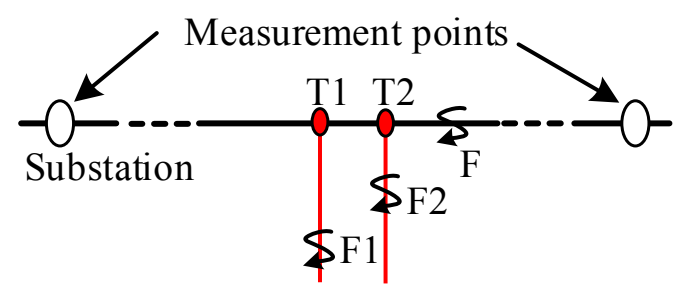

Fig. 2: Double-end method with faults on the main feeder and lateral

\section{Combined double-end and single-end method}

To distinguish between faults on the lateral and faults at the lateral tapping point, the measurements at the substation are used to calculate the voltages and currents at the lateral tapping point (using ladder power flow). Then, by using these calculated signals, a single-end method is applied on the suspected lateral and there are two possibilities for the output. The first one is a sensible distance based on the fault location on the lateral which will ensure that the correct location is on the lateral and the method continues to find the exact location. The second is a very small fault distance, ideally zero, which indicates that the fault exists at the lateral start point which is the tapping point. The following flow chart shows how the 
combined method works. The concept for the single-end method is described in the next subsection.

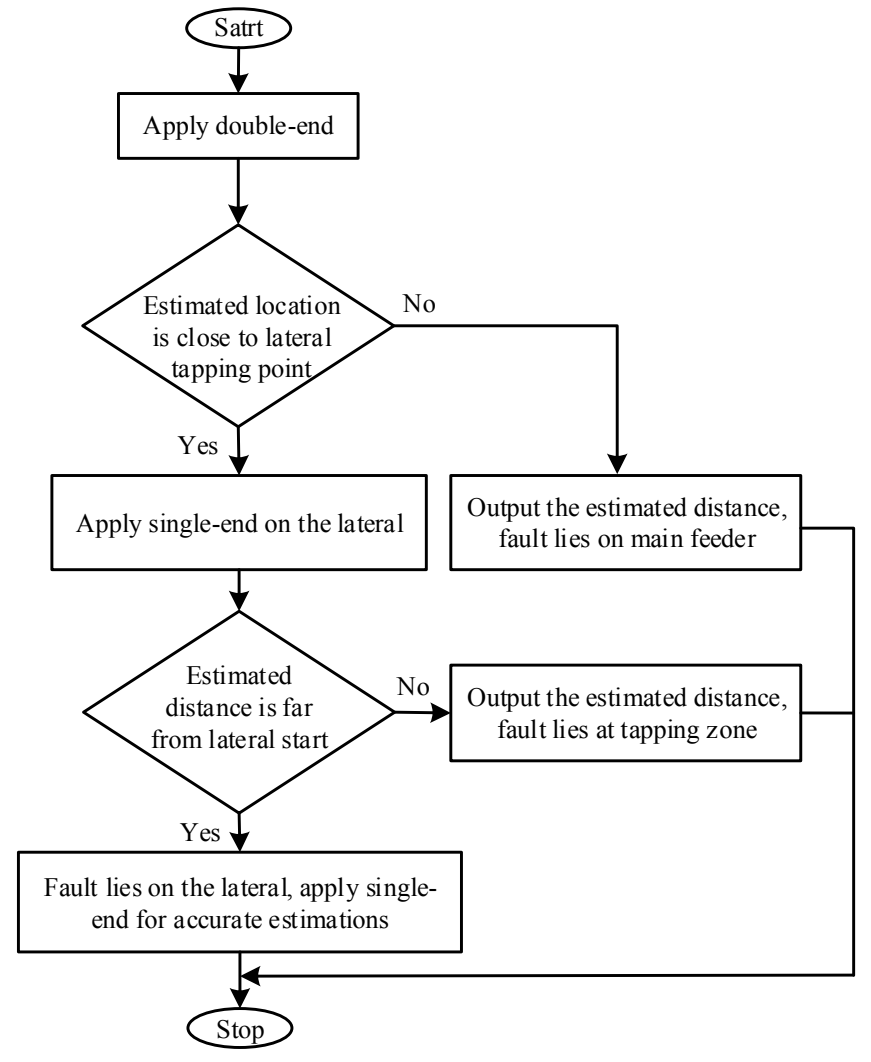

Fig. 3: Flowchart for combined double and single-end method

\subsection{Single-end method derivation}

Assuming voltages and currents are available from one side only, an iterative approach would be necessary to find the fault location. Similar to the double-end method, the fault is always assumed to be in the first section of the lateral, then moved forward if the estimated distance is greater than the total length of the assumed fault section. Consider Fig. 4, where a fault occurs at distance $\mathrm{x}$ from node $\mathrm{S}$, where voltages and currents at $S$ are obtained from measurements at the substation. The whole system beyond the assumed fault section is represented by an equivalent impedance $Z_{\text {th }}$. The fault is represented as a step voltage source $V_{\text {fault }}$ with a step change at the fault instant equal and opposite to the pre-fault voltage at point $f[14,18]$. The pre-fault voltage at point $f$ is calculated from the pre-fault voltage at $S$ using (2). The created $\mathrm{V}_{\text {fault }}$ is defined by (3).

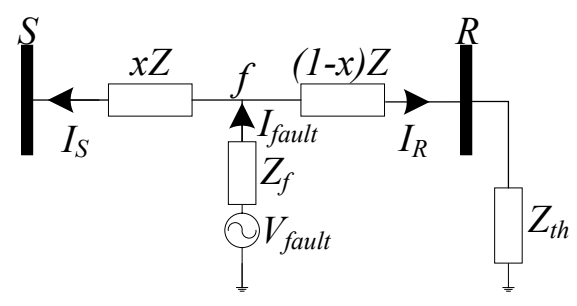

Fig. 4: Single-end method equivalent circuit

$$
\begin{gathered}
V_{\text {pre_f }}=V_{\text {pre_s }}+x Z I_{\text {pre_S }} \text { } \\
V_{\text {fault }}=\left\{\begin{array}{cl}
V_{\text {pre_f }} & \text { time }<\text { faultinstant } \\
0 & \text { time }>\text { faultinstant }
\end{array}\right.
\end{gathered}
$$

where $\mathrm{V}_{\text {pre_s }}$ and $\mathrm{I}_{\text {pre_s }}$ are steady state pre-fault voltage and current at node $\mathrm{S}$.

Over the frequency range of interest, the fault distance is calculated using the following:

$$
\begin{gathered}
V_{f}=V_{S}+x Z I_{S} \\
I_{R}=V_{f} /\left((1-x) Z+Z_{t h}\right) \\
Z_{f}=\left(V_{\text {fault }}-V_{f}\right) /\left(I_{S}+I_{R}\right)
\end{gathered}
$$

Equations (2) to (6), require the fault distance $\mathrm{x}$ to be known. Hence, an initial value of $\mathrm{x}$ could be used e.g. $0 \mathrm{pu}$ or $0.5 \mathrm{pu}$. After that, a new value for the fault distance is calculated iteratively using (7).

$$
x\left(Z I_{S}\right)=V_{\text {fault }}-V_{S}-Z_{f}\left(I_{S}+I_{R}\right)
$$

If the new value is greater than $1 \mathrm{pu}$, the algorithm should move forward to next sections. Otherwise, in the same section, repeat the method using (2) to (7) with the newest estimated distance until an accepted difference between two iterations is obtained.

\section{Test system}

In order to evaluate the validity of the method, the IEEE 34 nodes system is used and simulated using Matlab/Simulink [19]. This system has been widely used in earlier fault location studies. It consists of a main feeder with different conductor sizes, single phase and three phase lateral and also unbalanced loading. In this study, the loads are considered as constant impedances. The data has been captured for $15 \mathrm{~ms}$ before and after the fault instant at a sampling rate of $20 \mathrm{kHz}$. The two measurement points lie at nodes 800 and 848 . Single line to ground (SLG), line to line (LL) and three-phase (3ph) faults are examined to check accuracy against both ground and phase faults. Different values for both the fault resistance and the fault inception angle are used. The evaluation begins with the double-end method for faults along the feeder extending between nodes 800 and 848 . Then, the performance of the combined method for faults along laterals is illustrated followed by a sample of results for the single-end method when applied on the lateral.

\subsection{Results for double-end method only}

The three fault types are simulated at nine locations along the feeder from node 800 to node 848 . The error in estimated distance measured in meters is defined by (8)

error $=$ estimated distance - actual distance 


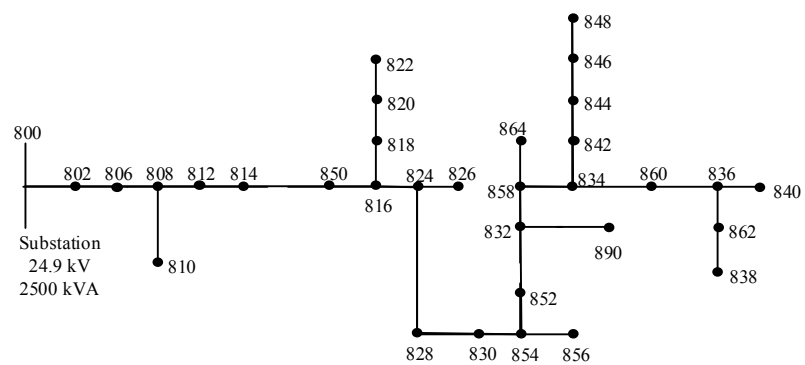

Fig. 5: IEEE 34 nodes system

\subsubsection{Effect of fault resistance}

Four different fault resistance values which are $0.01,1,10$ and $100 \Omega$ are considered. The error in the estimated distance versus the actual fault location for the three fault types is shown in Fig. 6, Fig. 7 and Fig. 8. The three figures ensure the robustness of the method against the fault resistance value where the error for different cases is quite close. Also, the maximum absolute error is less than $80 \mathrm{~m}$ (noting the whole system represents a $58 \mathrm{~km}$ length of line)

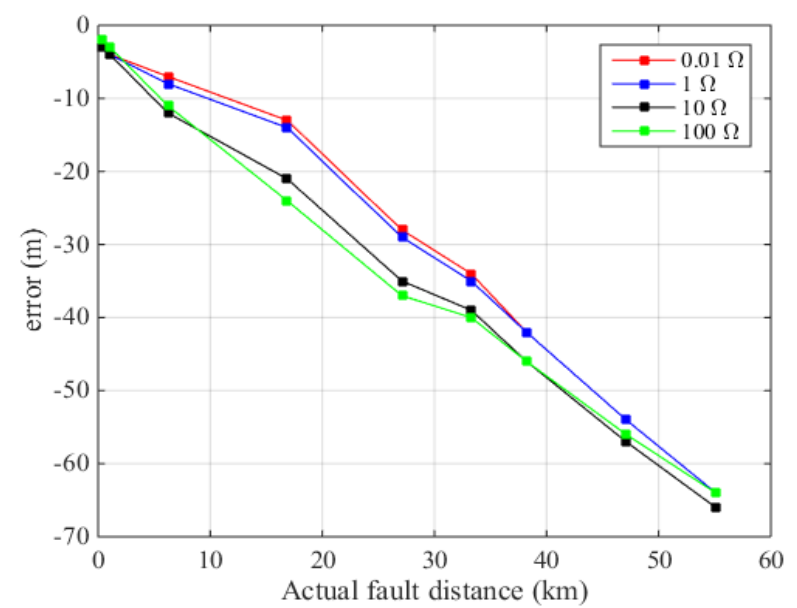

Fig. 6: Error in estimated distance for DEM for SLG fault for different fault resistance values

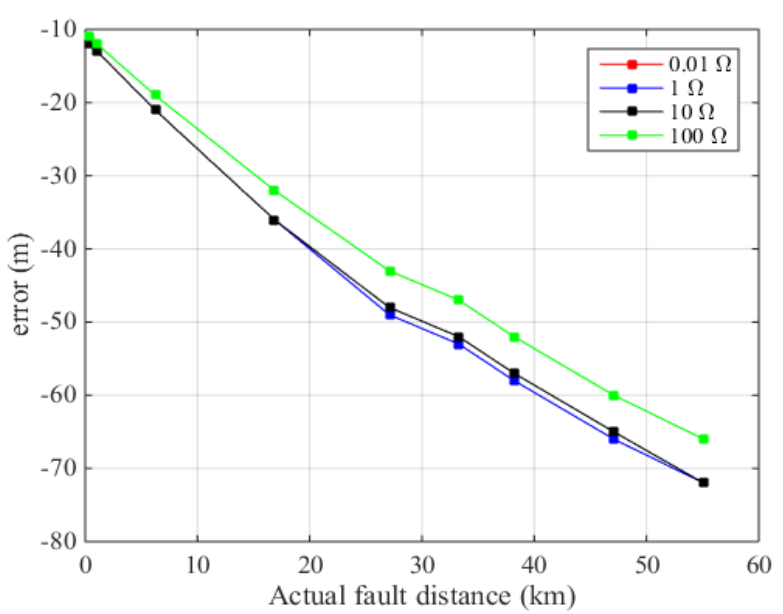

Fig. 7: Error in estimated distance for DEM for LL fault for different fault resistance values

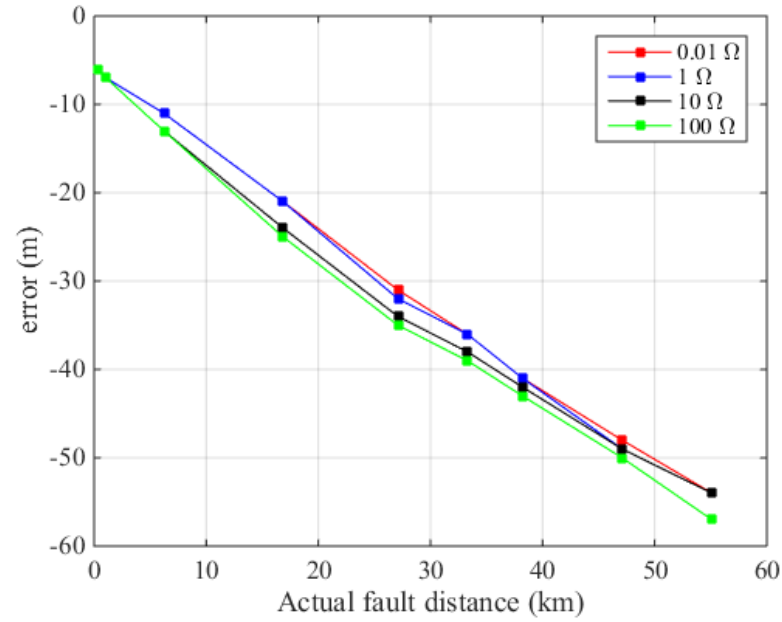

Fig. 8: Error in estimated distance for DEM for $3 \mathrm{ph}$ fault at different fault resistance values

\subsubsection{Effect of fault inception angle}

The method has been checked against a variety of fault inception angles (i.e. the point in supply voltage waveform when the fault occurs). The following figures illustrate the error for SLG, LL and 3ph faults. With a change in the fault inception angle, it is expected that the transient magnitude would change (especially for $0^{\circ}$ ). However, the method shows an accurate estimation for different cases as shown in Fig 911.

\subsection{Combined method performance}

Several faults under different operating conditions are simulated at lateral tapping points and along the laterals to check the performance of the combined method. Table 1 presents a sample of the test cases and the corresponding performance of the combined method. In this table, the actual distance is given as the sum of two values that represent the distance on the main feeder and on the lateral respectively.

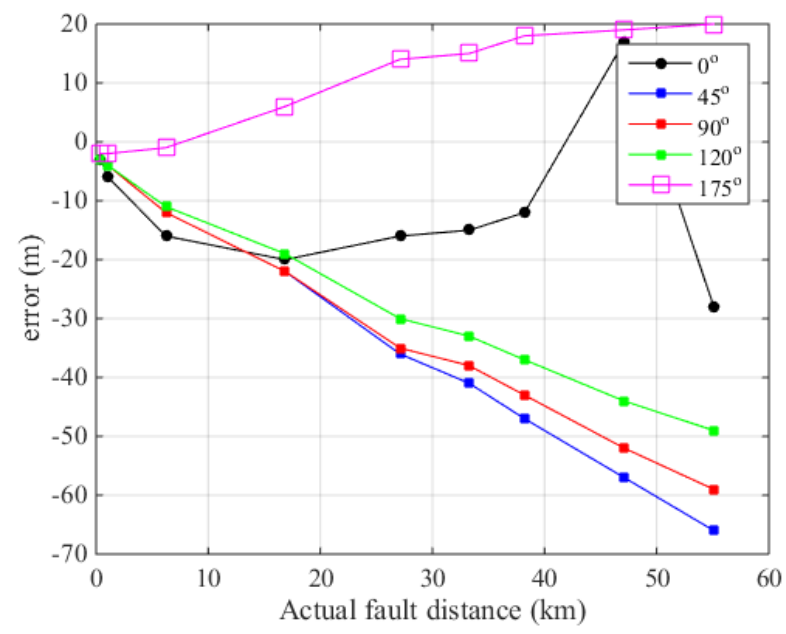

Fig.9: Error in estimated distance for DEM for SLG fault at different fault inception angles 


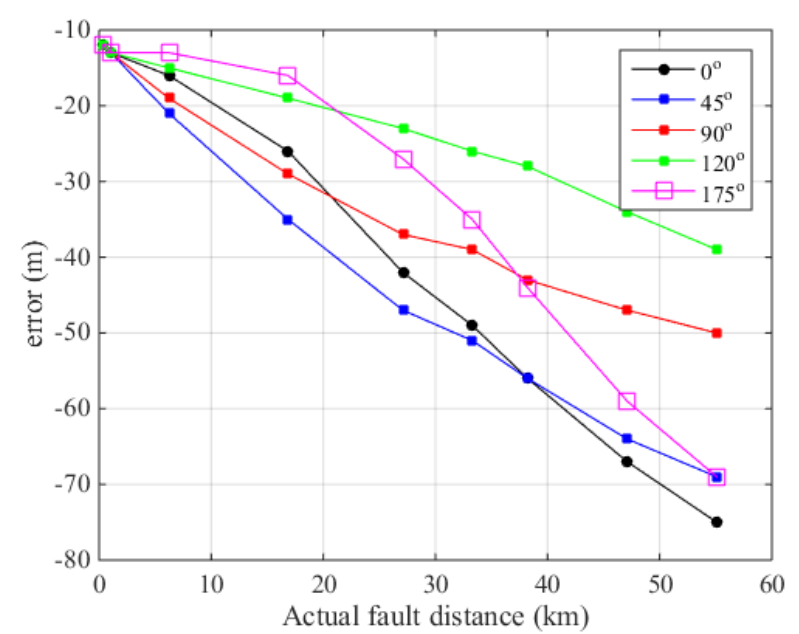

Fig. 10: Error in estimated distance for DEM for LL fault at different fault inception angles

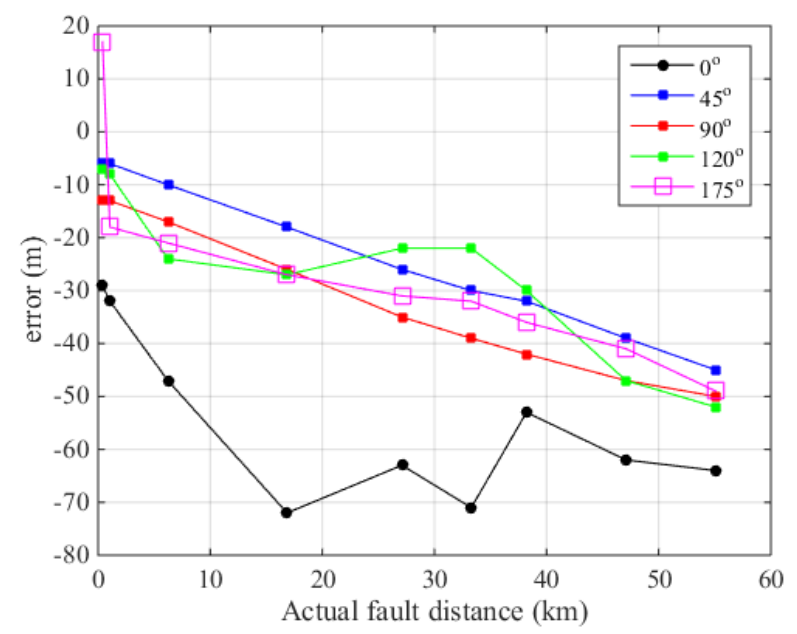

Fig. 11: Error in estimated distance for DEM for 3ph fault at different fault inception angles

\begin{tabular}{|c|c|c|c|c|}
\hline \multirow{2}{*}{$\begin{array}{c}\text { Tap } \\
\text { point }\end{array}$} & \multirow{2}{*}{$\begin{array}{c}\text { Fault } \\
\text { location }\end{array}$} & \multirow{2}{*}{$\begin{array}{c}\text { Actual } \\
\text { distance }(\mathrm{m})\end{array}$} & \multicolumn{2}{|c|}{ Estimated distance $(\mathrm{m})$} \\
\cline { 4 - 5 } & & Double-end & Single-end \\
\hline 808 & On lateral & $11137+1000$ & 11115 & 980 \\
\hline 808 & Tap point & $11137+0$ & 11115 & -18 \\
\hline 832 & On lateral & $52711+1000$ & 52662 & 957 \\
\hline 832 & Tap point & $52711+0$ & 52662 & -80 \\
\hline
\end{tabular}

Table 1: Combined method performance test

As seen from the table, the double end method provides one estimation and does not differentiate between faults at the tap and on the lateral. Single-end results give a small estimated distance in case of faults at the tap and a sensible distance for faults on the laterals.

\subsection{Results for single-end method}

To check the accuracy of the single-end method, it has been applied on the lateral at node 816. This lateral is a single phase lateral with a total length of about $19 \mathrm{~km}$. A SLG fault with different fault resistance values and a fault inception angle of $30^{\circ}$ is simulated at different locations on the lateral. The accuracy of the method is shown in Fig 12.

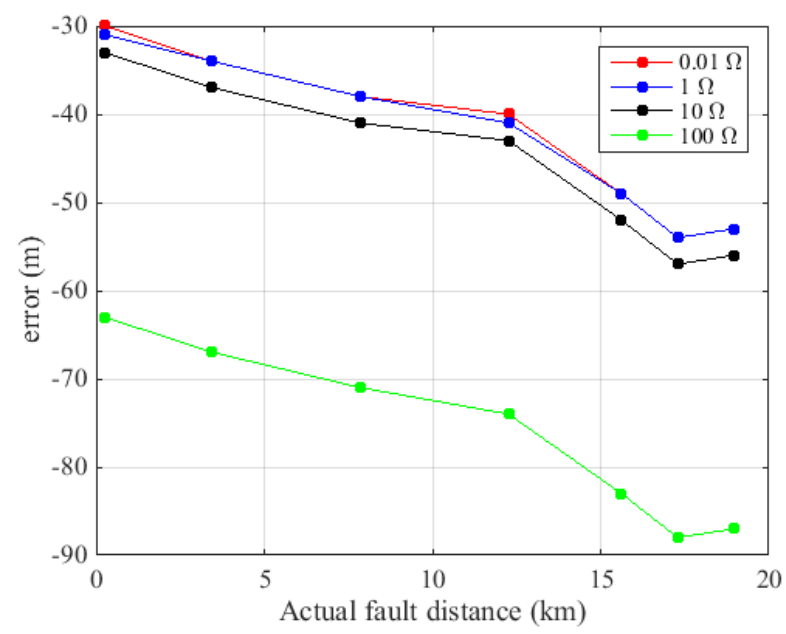

Fig. 12: Error in estimated distance for faults along lateral using SEM

Based on the previous evaluation under the test conditions, the maximum absolute error does not exceed $90 \mathrm{~m}$ for a system that has a total main feeder length of $58 \mathrm{~km}$ and laterals with a total length of $35 \mathrm{~km}$.

\section{Conclusions}

A combined double and single end impedance based fault locator has been presented. The method requires a short window of data for processing and depends on the high frequency components generated by the fault. The double-end algorithm provided a single solution wherever the fault location to be. The single-end has been employed to locate faults along laterals. The algorithm has been tested against single line to ground, line to line and three phase faults at different fault resistance values and fault inception angles. The results indicate a high accuracy with a maximum absolute error of $90 \mathrm{~m}$ for the IEEE 34 nodes system that has a longest feeder of $58 \mathrm{~km}$ and $35 \mathrm{~km}$ aggregated laterals. The results are encouraging for the real implementation of the proposed method.

\section{Acknowledgements}

This work was supported by the Egyptian Governmentministry of higher education (cultural affairs and missions sector) and the British Council through Newton-Mosharafa fund.

\section{References}

[1] M. M. Saha, J. Izykowski, and E. Rosolowski, Fault location on power networks: Springer, 2010. 
[2] L. Philipson and H. L. Willis, Understanding electric utilities and de-reguration, 2nd ed.: Taylor \& Francis Group, 2006.

[3] M. Kezunovic, "Smart Fault Location for Smart Grids," IEEE Transactions on Smart Grid, vol. 2, pp. 11-22, 2011.

[4] M. Dragomir, A. Miron, M. Istrate, and A. Dragomir, "A review of impedance-based fault location approaches for transmission lines," in 2014 International Conference and Exposition on Electrical and Power Engineering (EPE), 2014, pp. 1021-1024.

[5] F. M. Abo-Shady, M. A. Alaam, and A. M. Azmy, "Impedance-based fault location technique for distribution systems in presence of distributed generation," in 2013 IEEE International Conference on Smart Energy Grid Engineering (SEGE), 2013, pp. 1-6.

[6] Y. Li, H. Gao, Q. Du, X. Qi, Q. Pang, and G. Zhu, "A review of single-phase-to-ground fault location methods in distribution networks," in 2011 4th International Conference on Electric Utility Deregulation and Restructuring and Power Technologies (DRPT), 2011, pp. 938-943.

[7] M. Abad, M. García-Gracia, N. E. Halabi, and D. L. Andía, "Network impulse response based-on fault location method for fault location in power distribution systems," IET Generation, Transmission \& Distribution, vol. 10, pp. 3962-3970, 2016.

[8] A. Dwivedi and $\mathrm{X}$. Yu, "Fault location in radial distribution lines using travelling waves and network theory," in 2011 IEEE International Symposium on Industrial Electronics, 2011, pp. 1051-1056.

[9] C. Apisit, C. Positharn, and A. Ngaopitakkul, "Discrete wavelet transform and probabilistic neural network algorithm for fault location in underground cable," in 2012 International conference on Fuzzy Theory and Its Applications (iFUZZY2012), 2012, pp. 154-157.

[10] Y. Gong and A. Guzman, "Integrated Fault Location System for Power Distribution Feeders," IEEE Transactions on Industry Applications, vol. 49, pp. 1071-1078, 2013.

[11] J. H. Teng, W. H. Huang, and S. W. Luan, "Automatic and Fast Faulted Line-Section Location Method for Distribution Systems Based on Fault Indicators," IEEE Transactions on Power Systems, vol. 29, pp. 1653-1662, 2014.

[12] P. C. Chen, V. Malbasa, and M. Kezunovic, "Locating sub-cycle faults in distribution network applying halfcycle DFT method," in T\&D Conference and Exposition, 2014 IEEE PES, 2014, pp. 1-5.

[13] K. Jia, D. W. P. Thomas, and M. Sumner, "A New double-ended fault-location scheme for utilization in integrated power systems," IEEE Transactions on Power Delivery, vol. 28, pp. 594-603, 2013.

[14] K. Jia, T. Bi, Z. Ren, D. Thomas, and M. Sumner, "High frequency impedance based fault location in distribution system with DGs," IEEE Transactions on Smart Grid, vol. PP, pp. 1-1, 2016.

[15] J. Ren, S. S. Venkata, and E. Sortomme, "An Accurate Synchrophasor Based Fault Location Method for
Emerging Distribution Systems," IEEE Transactions on Power Delivery, vol. 29, pp. 297-298, 2014.

[16] J. Ramirez-Ramirez, J. Mora-Florez, and C. GrajalesEspinal, "Fault location method based on two end measurements at the power distribution system," in Circuits \& Systems (LASCAS), 2015 IEEE 6th Latin American Symposium on, 2015, pp. 1-4.

[17] W. H. Kersting, Distribution system modeling and analysis, 2 ed.: Taylor \& Francis Group, Boca Raton, 2007.

[18] K. Jia, D. Thomas, and M. Sumner, "A New SingleEnded Fault-Location Scheme for Utilization in an Integrated Power System," IEEE Transactions on Power Delivery, vol. 28, pp. 38-46, 2013.

[19] IEEE PES Distribution System Analysis Subcommittee, 34-bus feeder [Online]. Available: http://ewh.ieee.org/soc/pes/dsacom/testfeeders/index.ht $\mathrm{ml}$ 